\title{
Perceived Blur in Amblyopia
}

\author{
Anita J. Simmers, Peter J. Bex, and Robert F. Hess
}

Purpose. The well-documented fact that visual acuity and contrast sensitivity in amblyopia are attenuated at high spatial frequencies predicts that amblyopes should perceive objects as blurred, because they do not have the high spatial frequency information necessary to represent sharp edges adequately. In the current study, the representation of blur in amblyopia with blur-discrimination and blur-matching tasks was explored in a series of experiments.

MethoDs. Monocular blur-discrimination thresholds were measured in a spatial two-alternative forced-choice procedure. Observers were required to discriminate which edge (right or left) appeared to be the lesser blurred. Observers also interocularly matched edges that were identical with those used in the blur-discrimination tasks, with the exception that they were viewed dichoptically at all times.

RESULTS. Blur-discrimination thresholds were elevated in both the amblyopic and fellow fixing eyes but were within the normal range for interocular matching thresholds.

Conclusions. The results suggest that blur is veridically represented in the amblyopic visual system. The surprising result is that all amblyopes, even those with the most severe visual loss, veridically matched all blurred edges, including the sharpest ones. This implies that amblyopes are able to represent levels of blur that are defined by spatial structure beyond their resolution limit. (Invest Ophthalmol Vis Sci. 2003;44:1395-1400) DOI:10.1167/iovs.02-0625

$\mathrm{T}^{\mathrm{s}}$ he visual disorder of amblyopia affects $3 \%$ to $4 \%$ of the population. Amblyopia is a developmental condition that is characterized by reduced vision in one eye due to the presence of a sensory impediment during visual development, such as strabismus (ocular misalignment) or anisometropia (unequal refractive error), occurring early in life. Most studies in both humans $^{1,2}$ and animals ${ }^{3,4}$ point toward a cortical locus for the processing deficit in amblyopia, revealing sensory deficits at the single-cell level ${ }^{5-9}$ that include reduced spatial resolution, reduced contrast sensitivity, and a reduced number of binocular cells.

It follows, from the reduced acuity and contrast sensitivity in amblyopia and from the now-accepted notion that this is due to a loss and/or reduced sensitivity of cells that carry high spatial frequency information, ${ }^{6,8,9}$ that the representation of edges and contours should be more blurred than in observers with higher acuity and normal contrast sensitivity. In other words, the expected finding would be that, as a consequence

From The Institute of Ophthalmology, University College London, London, United Kingdom; and McGill Vision Research, Department of Ophthalmology, McGill University, Montreal, Canada.

Supported by a Medical Research Council Fellowship (AJS) and by a Wellcome grant (PJB).

Submitted for publication June 25, 2002; revised September 12, 2002; accepted October 8, 2002.

Disclosure: A.J. Simmers, None; P.J. Bex, None; R.F. Hess, None

The publication costs of this article were defrayed in part by page charge payment. This article must therefore be marked "advertisement" in accordance with 18 U.S.C. $\$ 1734$ solely to indicate this fact.

Corresponding author: Anita J. Simmers, MRC Research Fellow, The Institute of Ophthalmology, University College London, London, UK EC1V 9EL; a.simmers@ucl.ac.uk. of the neural deficit, the amblyopic visual system exhibits an increased level of intrinsic blur (i.e., neural blur). However, although it is well documented that visual acuity and contrast sensitivity in amblyopia are attenuated at high spatial frequencies, anecdotally, amblyopes do not report that images appear blurred, and little quantitative information is available on how blurred the perception is in amblyopia.

Several studies have examined the perception of blur under conditions of impoverished spatial vision. Owing to the temporal integration of the visual system ${ }^{10,11}$ it might be expected that moving objects would appear blurred. However, they usually appear sharp, and this effect can be accounted for by the spatiotemporal orientation of the receptive fields of motion detectors. ${ }^{12}$ However, this account does not explain the observation that when a blurred object is set in motion, it can appear sharper than when it is static. ${ }^{13-15}$ Similarly, objects viewed in a peripheral visual field should appear blurred owing to the decline in acuity, ${ }^{16}$ but they usually appear quite sharp, a phenomenon termed "sharpness overconstancy." 17,18 These results require a spatial frequency and speed- ${ }^{15,19}$ or eccentricity-dependent contrast gain change, or a high-level interpretation of image structure. ${ }^{18,20}$ It is therefore possible that the sharp appearance of images that may be spatially misrepresented in the amblyopic visual system may also be the result of sharpness overconstancy. We explored this possibility by measuring the representation of blur in amblyopia with blur-discrimination and blur-matching tasks.

\section{Methods}

\section{Observers}

Six amblyopes, four with strabismus, two with anisometropia, and two with strabismus and anisometropia (mean age, $29.4 \pm 5.8$ years), were recruited for the study. A control group of three observers (mean age, $27 \pm 4.4$ years) were selected who had normal visual acuity and binocular vision. Viewing was monocular in all cases with the appropriate refractive correction. All experimental procedures adhered to the tenets of the Declaration of Helsinki, and informed consent was obtained once the nature and possible consequences of the experiment had been explained.

\section{Apparatus and Stimuli}

Stimuli were generated on a computer (Macintosh G4; Apple Computer, Cupertino, CA) using software adapted from VideoToolbox routines (http://www.vision.nyu.edu/developed by Dennis Pelli, New York University, New York, NY). ${ }^{21}$ Images were displayed on a multiscan gray-scale monitor (model 500PS Trintitron; Sony, Tokyo, Japan) at a frame rate of $75 \mathrm{~Hz}$ and a mean luminance of $50 \mathrm{~cd} / \mathrm{m}^{2}$. The luminance of the display was linearized to a pseudo 12-bit resolution with a video attenuator ${ }^{22}$ and calibrated with a photometer (Minolta, Ramsey, NJ). Pseudo 12-bit resolution in this case allows the presentation of $2^{8}$ monochrome levels of a possible $2^{12}$ levels. Images were presented in gray scale by amplifying the monochrome signal and driving the red, green, and blue guns equally. The display was $36^{\circ}$ horizontally ( 1152 pixels) by $27.2^{\circ}$ vertically ( 870 pixels) and was viewed monocularly in a dark room from a distance of $57 \mathrm{~cm}$.

\section{Experiment 1: Edge Blur Discrimination}

Blur-discrimination thresholds serve as an objective measure of the representation of blur in the visual system. We compared blur discrim- 
ination for high-contrast edges between amblyopes (see Table 1 for clinical details) and normal observers.

\section{Procedure}

Blur-discrimination thresholds were measured in a spatial two-alternative force-choice procedure. Static, blurred edges were presented in two vertically oriented elongated bars $\left(2 \times 6.1^{\circ}\right) 2^{\circ}$ either side of a central fixation cross (Fig. 1a). The luminance profiles of the blurred edge were cumulative Gaussian. The standard deviation of the reference blurred edge $(b)$ was fixed at $1.88,3.75,7.5,15,30$, or 60 minutes of arc (minarc), randomly interleaved within a test session. The standard deviation of the comparison blurred edge was always greater than that of the reference, by $\Delta b$, under computer control with a QUEST staircase $^{23}$ designed to concentrate observations at a $75 \%$ correct level. To prevent observers from using artifactual cues to blur extent, such as contrast, vernier alignment, or the extent of light or dark regions, we randomized several variables: The positions of the edges within the rectangular windows were randomized, the polarity between standard and reference edges was reversed, and the Michelson contrast of the edges was $75 \% \pm 0 \%$ to $\pm 10 \%$ at random. The edges were presented for 1 second, with onset and offset smoothed by a raised cosine temporal envelope over $50 \mathrm{~ms}$.

For a short interval ( 1 minute) before the start of each experiment, the screen was a blank mean luminance field to ensure a constant state of adaptation. The subject was instructed to fixate the cross and to identify which edge (right or left) appeared to be the lesser blurred. Auditory feedback was provided after incorrect responses. In those observers with amblyopia, measurements were repeated with both the amblyopic eye and nonamblyopic eye in random order. Each test contained 32 trials randomly interleaved for each of the six levels of blur and was repeated a minimum of four times by each observer, randomly interleaved with blur-matching conditions, which will be described later. The raw data across all tests for each condition for each observer were combined and were fitted with cumulative normal psychometric functions by a least $\chi^{2}$ fit. From this fit, the thresholds and $95 \%$ confidence limits were estimated at the $75 \%$ correct point.

The changes in the appearance of the stimuli are illustrated in a video at the following Web site: http://www.ucl.ac.uk/ $\sim$ smgxpbe/ amblyopia.html. The reader is encouraged to move the frame-slider by hand to see how the stimulus varies from minimal to maximal blur.

TaBle 1. Clinical Characteristics of the Subjects with Amblyopia

\begin{tabular}{|c|c|c|c|}
\hline Subject & Visual Acuity & $\begin{array}{c}\text { Spectacle } \\
\text { Prescription }\end{array}$ & $\begin{array}{c}\text { Ocular } \\
\text { Alignment }\end{array}$ \\
\hline \multirow[t]{2}{*}{0} & RE $6 / 5$ & None & L SOT \\
\hline & LE $6 / 15$ & & $10 \Delta$ \\
\hline \multirow[t]{2}{*}{$\Delta$} & RE $1 / 30$ & None & L XOT \\
\hline & LE $6 / 5$ & & $15 \Delta$ \\
\hline \multirow[t]{2}{*}{$\square$} & RE $1 / 60$ & None & R SOT \\
\hline & LE $6 / 6$ & & $20 \Delta$ \\
\hline \multirow[t]{2}{*}{$\diamond$} & RE 6/6 & None & L SOT \\
\hline & LE $6 / 38$ & & $20 \Delta$ \\
\hline \multirow[t]{2}{*}{$\square$} & RE $6 / 6$ & $\mathrm{RE}+4.00 /-1.00 \times 170$ & L XOT \\
\hline & LE $6 / 38$ & $\mathrm{LE}+6.00 /-1.75 \times 177$ & $14 \Delta$ \\
\hline \multirow[t]{2}{*}{0} & RE 6/6 & RE plano & L XOT \\
\hline & LE $6 / 15$ & $\mathrm{LE}+3.25 \times 90$ & $8 \Delta$ \\
\hline \multirow[t]{2}{*}{$\diamond$} & $\mathrm{RE} 6 / 5$ & RE plano & Straight \\
\hline & LE $6 / 24$ & $\mathrm{LE}+2.50 \mathrm{DS}$ & \\
\hline \multirow[t]{2}{*}{$\Delta$} & RE $6 / 5$ & RE plano & Straight \\
\hline & LE $6 / 24$ & $\mathrm{LE}+3.25 /+1.00 \times 90$ & \\
\hline
\end{tabular}

Filled symbols correspond to individual strabismic, open symbols to either strabismic anisometropes or anisometropic amblyopes. Symbols correspond to symbols in the figures. SOT, convergent strabismus; XOT, divergent strabismus.
A
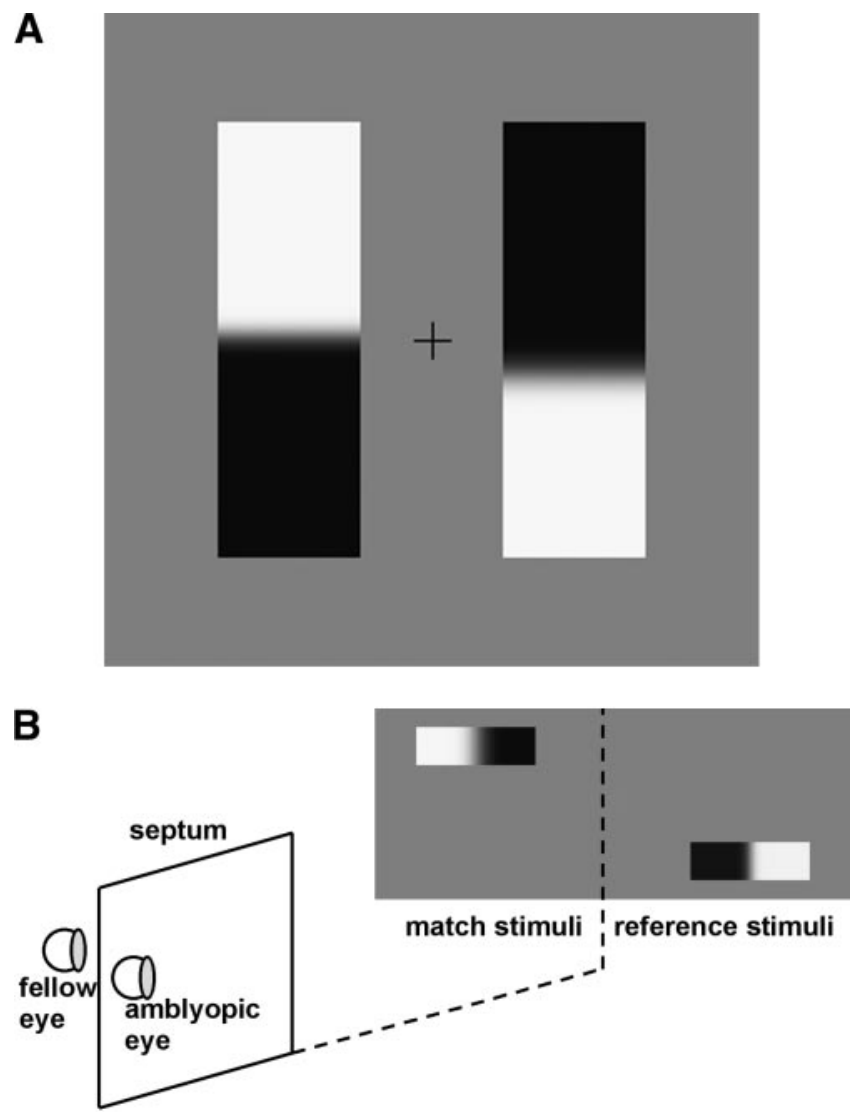

Figure 1. Illustration of the two stimulus arrangements used in the study for (a) blur discrimination and (b) interocular blur matching.

\section{Experiment 2: Interocular Matching}

To assess the degree of perceived blur experienced by the amblyopic visual system, we asked observers to match the blur of an edge seen only by the amblyopic eye with that of an edge seen only by the fellow eye. An edge stimuli with fixed degrees of Gaussian blur was presented to the amblyopic eye and a similar stimulus was presented to the normal eye, but its Gaussian blur was adjusted by the observer, to obtain a perceptual match. In view of the resolution and contrast sensitivity deficits known to characterize amblyopia ${ }^{24,25}$ the expectation is that a relatively sharp edge that is viewed by the amblyopic eye would appear somewhat blurred and therefore be matched with a relatively blurred edge viewed through the fellow eye

\section{Procedure}

Stimuli were identical with those used in blur-discrimination tasks, with the exception that they were viewed dichoptically, by means of a septum at all times. The rectangular windows were oriented horizontally with a horizontal offset of $2^{\circ}$, so that they appeared above and below the fixation cross, to prevent one of the edges from being suppressed by the amblyopic eye and to eliminate the risk of the two patterns fusing. An illustration of the stimuli is shown in Figure 1b. The standard deviations of the blur of the reference edges were the same as in the blur-discrimination conditions $\left(\sigma_{\mathrm{blur}}=1.88,3.75,7.5,15,30\right.$, or 60 minarc) randomly interleaved on a run. The standard deviation of the blur of the match edge was initialized with a random value between 0 and 90 minarc at the start of each trial and was then under the observer's control. The reference edge was presented to the amblyopic eye or was randomly assigned to the left or right eye of normalvision observers. Again, after a short interval to allow the subject to adapt to the darkened room and to ensure that the two eyes were correctly dissociated by the septum, observers were instructed to 
adjust the blur of the match edge so that it was equal to that of the reference edge by pressing one of two mouse buttons that increased or decreased the blur standard deviation of the match edge. Pressing a third mouse button indicated a satisfactory match and initiated the next trial. Adjustment time was unrestricted but was not usually more than 2 seconds. All observers matched each baseline blur level at least 15 times.

\section{Results}

\section{Experiment 1}

Figure 2 shows blur-discrimination thresholds $(\Delta b)$ as a function of baseline blur $(b)$. For all observers, blur-discrimination thresholds showed a characteristic dipper function. ${ }^{26,27}$

Blur-discrimination thresholds showed a clear tendency to be elevated in both the amblyopic and nonamblyopic fellow eye (Fig. 3) of the amblyopes. To investigate whether there were any statistically significant differences in the patterns of blur-discrimination deficits in the amblyopic observers, an analysis of variance (ANOVA) was performed on the data. A significant difference was revealed between subject groups, normal versus amblyopic versus fellow-eye $(d f=2 ; \mathrm{F}=17.34 ; P=$ $0.0001)$, and between the baseline level of blur ( $d f=5 ; \mathrm{F}=$ 45.588; $P=0.0001$ ). A significant interaction was also revealed between subject group and overall blur-discrimination deficit ( $d f=10 ; \mathrm{F}=2.442 ; P=0.01$ ). The ratio of normal to amblyopic eye performance was found to be $1: 3.57 \pm 1.17$ and that of normal compared with the fellow eye 1:2.04 \pm 0.94 . This difference in sensitivity to blur between subject groups is therefore manifested by a vertical shift in thresholds and thus appears multiplicative in nature, differing by up to a factor of 3 .

We fitted these data with a simple model based on the idea that at low levels of stimulus blur, discrimination is limited by intrinsic blur in the observers' visual system, whereas at high levels, performance is limited by the external blur in stimulus. The inflection point of the dipper function represents the equivalent intrinsic blur of the visual system and reflects constraints imposed by both optical and neural blur. At higher

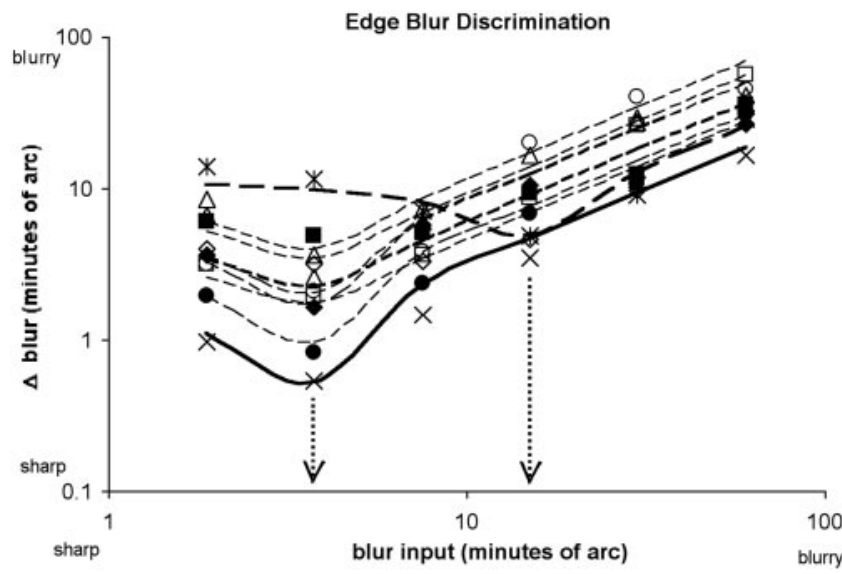

FIGURE 2. Edge blur-discrimination thresholds plotted as a function of baseline blur. Thresholds for individual amblyopes are plotted in different symbols (see corresponding symbols in Table 1 for subject details). $(X)$ Mean of three normal observers. $(\boldsymbol{X})$ One normal-vision observer with optical blur. The effects in the two subject groups are essentially the same with a level of intrinsic blur estimated at 3 minarc (indicated by the leftmost downward arrow). For blur extents greater than 3 minarc the data follow a power law. The addition of dioptric blur (*) shows an increase in intrinsic levels of blur (indicated by the rightmost downward arrow). Each estimate of threshold was based on at least four separate QUEST determinations (128 observations per point).

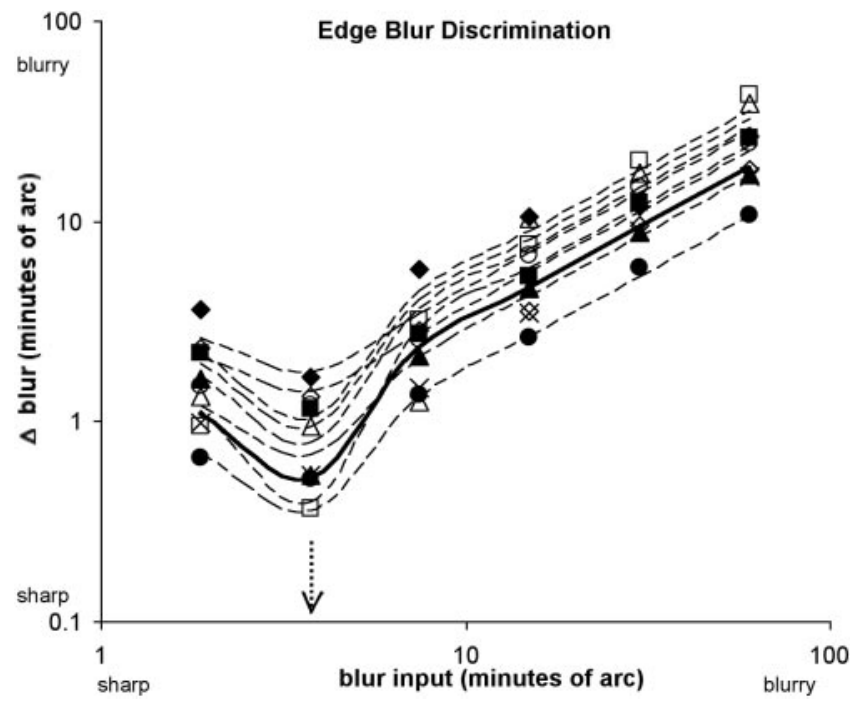

Figure 3. As in Figure 2, but for the nonamblyopic fellow eye.

levels of blur, discrimination is limited by extrinsic blur, and performance is according to Weber's law. No significant difference in intrinsic levels of blur was evident between normal and amblyopic $(P=0.97)$, amblyopic and nonamblyopic fellow eye $(P=0.66)$, or normal and nonamblyopic fellow eye $(P=0.8)$. No correlation was found between intrinsic levels of blur and the severity of amblyopia $(P>0.05)$.

If discrimination were selectively impaired for sharp edges, then the level of intrinsic blur would increase in the amblyopic group as gauged by a lateral shift in the inflection point of the dipper function. Figures 2 and 3 clearly show, in all observers, discrimination thresholds increasing at levels of blur approximating 3 to 4 minarc $(3.93 \pm 0.76)$, implying similar levels of intrinsic blur in the amblyopic visual system. Indeed, amblyopia cannot be mimicked by simply raising levels of blur, Figure 2 also shows the results for normal observer AJS (open circles) of artificially blurring with a +3.00 diopter sphere (DS). The function is now not only multiplicative in nature (i.e., reduced sensitivity, or vertical shift in the function) but also shows an additive error (i.e., representing increased levels of intrinsic blur within the observer's visual system, or shifting the minimum to the right). At levels of stimulus blur much larger than the minimum, thresholds for any amount of Gaussian blur are essentially identical with those obtained without dioptric blur.

Figure 4 shows the luminance profile of two Gaussian edges that can be reliably discriminated by an amblyopic observer: The dark line represents the difference in spatial profiles between these two edges. We used a Fourier transform of this difference to reveal the spatial frequencies that were used by each observer to support blur discrimination. Figure 5 shows a plot of the peak spatial frequency of the difference that is used by each observer to discriminate blurred edges at each standard deviation. At high levels of edge blur, normal observers and amblyopes used the same spatial frequencies to discriminate the edges. However, at lower levels of edge blur, discrimination was based on much higher spatial frequencies in observers with normal vision than in those with amblyopia. This shift to lower spatial frequencies in amblyopic observers is consistent with the reduced contrast sensitivity at higher spatial frequencies in these observers.

\section{Experiment 2}

Figure 6 shows the blur of a comparison edge viewed with the normal eye that matched that of a reference edge viewed with 


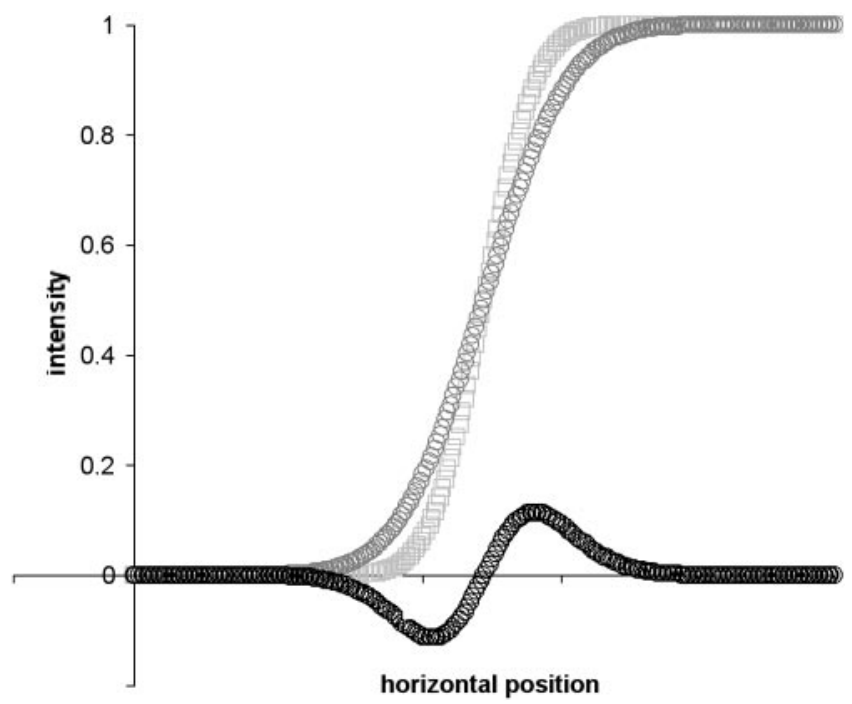

FigURE 4. Luminance profile of two typical Gaussian edges that can be reliably discriminated by an amblyope, the dark line represents the difference in spatial profiles between the two edges. A fast Fourier transform of the difference image was used to determine the spatial frequencies used by subjects to perform the discrimination.

the amblyopic eye as a function of the blur of the reference edge. Normal observers viewed the reference and match edges with either the left or right eye at random, and all matches fell on the diagonal line corresponding to veridical perception (data not shown). The matches for the amblyopic group unexpectedly also fell on the diagonal line, indicating that all edges appeared equally blurred whether viewed through the amblyopic or the fellow eye.

For comparison, the blur-matching task was repeated by a normal observer uniocularly, optically blurred with a +3-D lens (Fig. 6, star symbol, dashed lines). Increasing the blur of an edge in this way selectively attenuates high-spatial-frequency structure, which elevates spatial acuity thresholds (star symbol on the $x$-axis) and lowers contrast sensitivity at mid high spatial

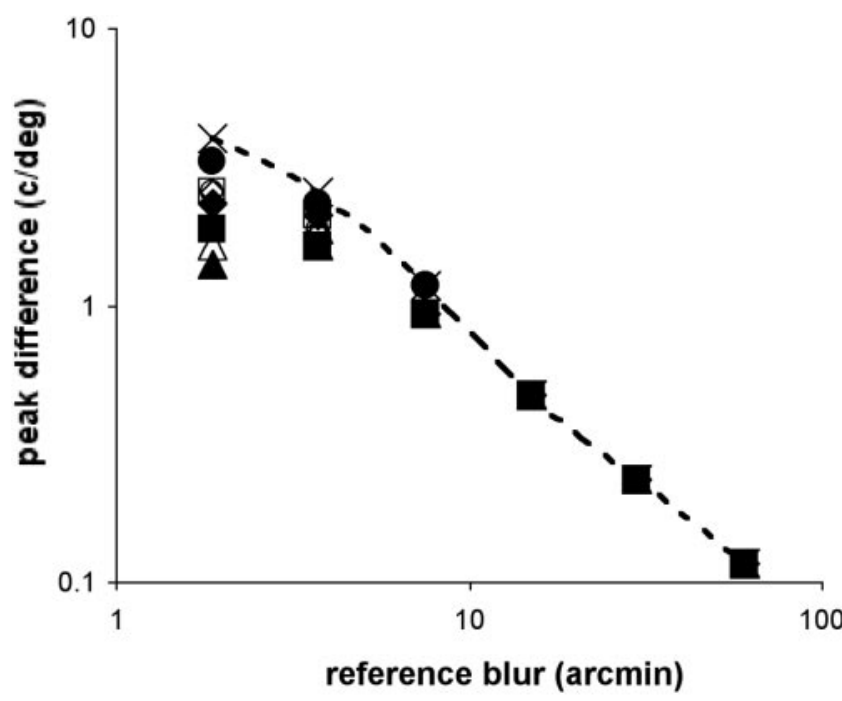

Figure 5. Peak spatial frequency that was used to discriminate blurred edges at each standard deviation. A Fourier transform of the difference in spatial profiles between the two edges reveals the spatial frequencies that support blur discrimination. $(X$, dashed line $)$ The normal observers; the remaining symbols are those of the individual amblyopes (Table 1).

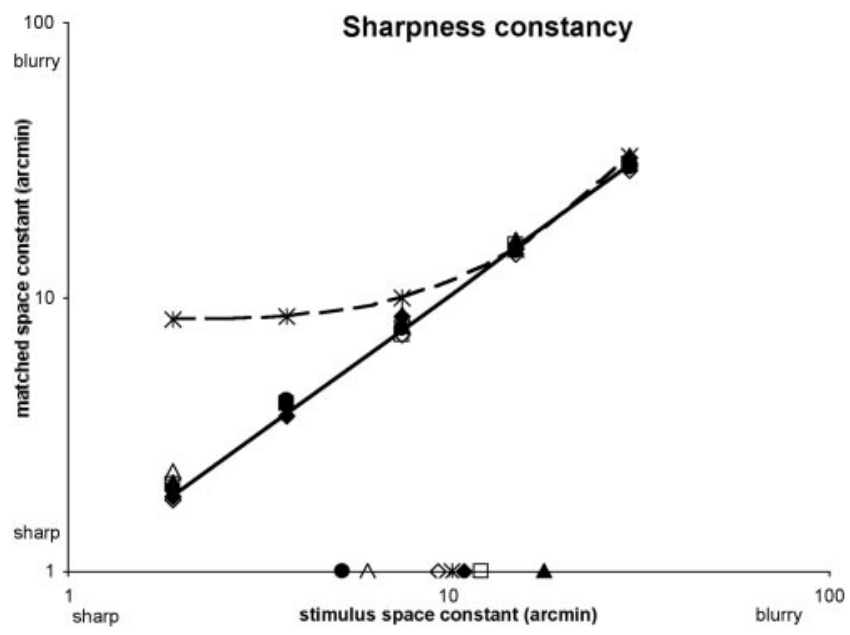

Figure 6. Matched blur as a function of stimulus blur in individual amblyopes and one normal observer with optical blur ( $\boldsymbol{*})$. Diagonal line shows where the means would fall if the observers showed perfect sharpness constancy. The axes are plotted so that sharpness decreases away from the origin. The individual resolution thresholds are also plotted on the $x$-axis, along with that of a normal-vision observer with optical blur ( $)$. Matching is veridical with all amblyopes. At low levels of reference blur the observer with optical blur matches a fairly sharp reference edge (viewed with the optically blurred eye) with a much more blurred match edge. However, at high levels of reference blur (which is relatively unaffected by the optical blur), matching is veridical.

frequencies, bringing them into line with the average acuity deficit for our amblyopes. However, blur-matching functions for a normal-vision observer with optical blur were quite unlike those of amblyopes. At low levels of reference blur, the observer matched a fairly sharp edge (that had been optically blurred) with a much more blurred match edge. However, at high levels of reference blur (which is relatively unaffected by the optical blur), matching was veridical. These results demonstrate the interdependence of stimulus blur and intrinsic blur attributable to the visual system and show that the spatial misrepresentation of amblyopia is not well characterized by increased blurring at the first stages of visual processing.

The individual resolution thresholds of the amblyopes are also plotted on the $x$-axis of Figure 6, along with that of the normal-vision observer with optical blur. For this observer, there was good agreement between perceived blur and resolution acuity with optical blur. However, amblyopes veridically matched levels of blur that were well beyond their resolution limits. More recently, we have verified (Hess RF, Simmers AJ, and Bex PJ, manuscript submitted) that this is the case for the different types of amblyopia, namely strabismic, anisometropic, and mixed strabismic-anisometropic.

The results of Experiment 1 show that blur-discrimination thresholds can also be slightly elevated in the fellow eye of some amblyopes. It is therefore possible that in these observers, veridical blur matches reflect matches between equally blurred inputs. However, veridical interocular blur matches were achieved by other amblyopes whose fellow eye blurdiscrimination thresholds were as low or lower than those of normal-vision observers. This explanation does not account for veridical blur matches in these subjects.

To calculate the sharpest edge that our amblyopes could in principle represent, we low-pass filtered a sharp edge to remove those spatial frequencies that were beyond their resolution limit. The standard deviation of the best-fitting cumulative Gaussian to this edge represents the sharpest edge that they could theoretically encode. The sharpest edge estimated in this 
way was $\sigma$ of 8 minarc, which is much more blurred than the edges that the amblyopes accurately matched. It appears that amblyopes can represent spatial frequency structure beyond their resolution limits.

These results demonstrate the perceptual consequences of an artificially induced intrinsic blur and the inappropriateness of such a model for the early stages of assessing in amblyopia.

\section{Discussion}

The results of experiment 1 show that blur discrimination in our amblyopes was generally noisier than in the normal-vision observers, but that the intrinsic blur was similar to levels recorded in normal-vision observers. Experiment 2 shows that the amblyopes, even those with severe cases, could veridically match the sharpness of edges, even when the spatial composition of these edges were outside their visible resolution range. This is a robust finding; a series of control experiments verified that this was true of edges of different contrasts and spatial extents (Hess RF, Simmers AJ, Bex PJ, manuscript submitted). Taken together, these results suggest that blur is veridically represented in the amblyopic visual system. Amblyopia may be characterized by reduced spatial acuity and contrast sensitivity but an amblyope's visual percept is not that of blur. This is surprising, because it does not follow on from the known contrast-sensitivity loss or indeed the restricted spatial range available. These results also raise interesting questions about the mechanism by which blur is represented in the normal visual system.

Consider the model by Watt and $\mathrm{Hess}^{28}$ that the visual system has an intrinsic error due to blur that is combined with any stimulus blur. Performance is limited by whichever is the greater source of uncertainty. When the stimulus blur is relatively large, sensitivity depends on the external blur of the stimulus, because intrinsic blur has little additive effect on the representation of blur. However, when stimulus blur is relatively small, performance is limited by intrinsic blur. If amblyopia were well-characterized by greater levels of intrinsic blur, the minimum point of blur-discrimination thresholds would be expected to shift to the right. At high levels of external blur, performance of both amblyopes and normal-vision observers is constrained by external sources of blur, and under these conditions, blur-discrimination thresholds should converge. In this study, amblyopes did not show increased levels of intrinsic (neural) blur, but showed a reduction in sensitivity. Because the discrimination region in amblyopia appears normal, this increase in threshold may be due to an increase in the uncertainty or error of the neural representations being discriminated. In a discrimination task, noise is the limiting factor, and the increased thresholds in the present study represent increased noise in the visual system. Although amblyopes had elevated blur-discrimination thresholds, indicative of a noisy visual system, they did not show increased levels of intrinsic blur as have been previously documented. ${ }^{29}$

The apparent blur of edges viewed with an amblyopic eye was assessed with an interocular blur-matching task over a range of blurs. We were not surprised that normal-vision observers matched blur veridically and that the results fell along a line of unity slope. Also when one normal-vision eye was optically blurred, perceived blur was consistent with the level of blur that this introduces. The surprising result is that all amblyopes, even those with the most severe visual loss, veridically matched all blurred edges, including the sharpest ones. This implies that amblyopes are able to represent levels of blur that are defined by spatial frequencies beyond their resolution limit. This was verified by convolving a sharp edge with the amblyope's resolution acuity.
The results of this study are difficult to reconcile with the literature on how the visual system processes blur. In general, manipulations that blur visual images increase blur-discrimination thresholds and increase blur-matching levels. This was demonstrated in the present study by optically blurring a normal-vision observer. However, previous studies of perceived blur under conditions of impoverished spatial visibility, such as at high speed ${ }^{13-15}$ or in the peripheral visual field, ${ }^{17,18}$ have shown that blurred edges are much sharper than expected, given the decline in sensitivity to high spatial frequencies under these conditions. These results require a spatial frequency and speed- ${ }^{15,19}$ or eccentricity-dependent contrast gain change, an active deblurring process. ${ }^{30}$ Alternatively, a sharp appearance of objects may be a default perception when the visual system does not have sufficient resolution or knowledge of the spatial structure of environment to encode the blur of an object veridically. ${ }^{18,20}$ In the latter case, information about the sharpness of objects is provided by static, foveal glances. Similar approaches may be able to account for the "sharpness overconstancy" observed in the current study in amblyopia. It is possible that amblyopes perceive sharp edges as sharp, even though their early visual representation is blurred due to filtering losses because of high-level compensation. To explain the present matching results, the compensation mechanism must have detailed knowledge of the resolution acuity and contrast sensitivity of lower visual processes. If this occurs at a binocular site then such information about the real sharpness of objects may be provided by the fellow eye.

It has become apparent in recent years that the perceptual difficulties experienced by amblyopes when using the amblyopic eye is due to a spatial rather than a contrast disturbance. Much of the recent work on amblyopia has centered on the perceptual deficit, in particular, the positional uncertainty amblyopes demonstrate in judging the relative position of a target with respect to a nearby reference. Amblyopes consistently show marked losses in spatial accuracy uncorrelated with either their contrast or acuity loss.

The consensus at present suggests these losses may be modeled in terms of either a distorted spatial representation in the cortex ${ }^{31,32}$ or as a result of a sparse cortical spatial sampling grain. ${ }^{33,34}$ With respect to findings in the present study, a neural undersampling hypothesis would predict an increase in blur-discrimination thresholds with an increase in intrinsic blur, but our data do not show these effects. Scrambling an image, however, may perturb spatial information, but a scrambled image may contain more information than an undersampled image because the global statistics of the visual scene are preserved and could, in principle, support a veridical global percept. Amblyopic observers were able to match edge blur at spatial frequencies beyond their resolution limit, and their intrinsic blur estimated from blur discrimination was the same for the amblyopic and the normal fellow eye. Our results therefore favor models of the spatial deficit in amblyopia that are based on spatial perturbations or scrambling, over models based on undersampling.

\section{References}

1. Hess RF, Baker CL. Assessment of retinal function in severely amblyopic individuals. Vision Res. 1984;24:1367-1376.

2. Hess RF, Baker CL, Verhoeve JN, Tulney KU, France TD. The pattern evoked electroretinogram: its variability in normals and its relationship to amblyopia. Invest Opbthalmol Vis Sci. 1985;26: $1610-1623$

3. Cleland BG, Crewther DP, Crewther SG, Mitchell DEM. Normality of spatial resolution of retinal ganglion cells in cats with strabismic amblyopia. J Pbysiol (Lond). 1982;326:235-249.

4. Crewther DP, Crewther SG, Cleland BG. Is the retina sensitive to the effects of prolonged blur? Exp Brain Res. 1985;58:427-434. 
5. Eggers HM, Blakemore C. Physiological basis of anisometropic amblyopia. Science. 1978;201:264-267.

6. Chino YM, Shansky MS, Jankowski WL, Banser FA. Effects of rearing kittens with convergent strabismus on development of receptive-field properties in striate cortex neurons. $J$ Neurophysiol. 1983;50:265-286.

7. Movhson AJ, Eggers HM, Gizzi MS, Hendrickson AE, Kiorpes L, Boothe RG. Effects of unilateral blur on the macaque's visual system. III. Physiological observations. J Neurosci. 1987;7:13401351.

8. Crewther DP, Crewther SG. Neural site of strabismic amblyopia in cats: spatial frequency deficit in primary cortical neurons. Exp Brain Res. 1990;79:615-622.

9. Kiorpes L, Kiper DC, O'Keefe LP, Cavanaugh JR, Movshon JA Neuronal correlates of amblyopia in the visual cortex of macaque monkeys with experimental strabismus and anisometropia. J Neurosci. 1998;18:6411-6424.

10. Barlow HB. Temporal and spatial summation in human vision at different background intensities. J Physiol. 1958;141:337-350.

11. Burr DC. Temporal summation of moving images by the human visual system. Proc R Soc Lond B Biol Sci. 1981;211:321-339.

12. Burr DC, Ross J. Visual processing of motion. Trends Neurosci. 1986;9:304-307.

13. Ramachandran VS, Madhusudhan V, Vidyasagar TR. Sharpness constancy during movement perception. Perception. 1974;3:97-98.

14. Bex PJ, Edgar GK, Smith AT. Sharpening of drifting, blurred images. Vision Res. 1995;35:2539-2546.

15. Hammett ST. Motion blur and motion sharpening in the human visual system. Vision Res. 1997;37:2505-2510.

16. Aubert H, Forster B. Beiträge zur Kenntniss des indirekte Sehens (I) Untersuchungen über den Raumsinn der Retina. Arch Ophthalmol. 1857;3:1-37.

17. Galvin SJ, Oshea RP, Squire AM, Govan DG. Sharpness overconstancy in peripheral vision. Vision Res. 1997;37:2035-2039.

18. Galvin SJ, O'Shea RP, Squire AM, Hailstone DS. Sharpness overconstancy: the roles of visibility and current context. Vision Res. 1999;39:2649-2657.
19. Hammett ST, Bex PJ. Motion sharpening: evidence for the addition of high spatial frequencies to the effective neural image. Vision Res. 1996;36:2729-2733.

20. Burr DC, Morgan MJ. Motion deblurring in human vision. Proc $R$ Soc Lond B Biol Sci. 1997;264:431-436.

21. Pelli DG. The VideoToolbox software for visual psychophysics: transforming numbers into movies. Spat Vis. 1997;10:437-442.

22. Pelli DG, Zhang L. Accurate control of contrast on microcomputer displays. Vision Res. 1991;31:1337-1350.

23. Watson AB, Pelli DG. QUEST: A Bayesian adaptive psychometric method. Percept Psychophys. 1983;33:113-120.

24. Hess RF. Contrast sensitivity assessment of functional amblyopia in humans. Trans Ophthalmol Soc UK. 1979;99:391-397.

25. Levi DM, Harwerth RS, Manny, RE. Suprathreshold spatial frequency detection and binocular interaction in strabismic and anisometropic amblyopia. Invest Ophthalmol Vis Sci. 1979;18:714725 .

26. Legge GE. A power law for contrast discrimination. Vision Res. $1981 ; 21: 457-467$

27. Watt RJ, Morgan MJ. Mechanisms responsible for the assessment of visual location: theory and evidence. Vision Res. 1983;23:97-109.

28. Watt RJ, Hess RF. Spatial information and uncertainty in anisometropic amblyopia. Vis Res. 1987;27:661-674.

29. Levi DM, Klein SA. Equivalent intrinsic blur in amblyopia. Vision Res. 1990;30:1995-2022.

30. Anderson CH, Van Essen DC. Shifter circuits: a computational strategy for dynamic aspects of visual processing. P Natl Acad Sci USA. 1987;84:6297-6301.

31. Hess RF, Campbell FW, Greenhalgh T. On the nature of the neural abnormality in human amblyopia; neural aberrations and neural sensitivity loss. Pflugers Arch. 1978;377:201-207.

32. Hess RF. Developmental sensory impairment: amblyopia or tarachopia? Hum Neurobiol. 1982;1:17-29.

33. Levi DM, Klein SA. Spatial localization in normal and amblyopic vision. Vision Res. 1983;23:1005-1017.

34. Levi DM, Klein SA, Aitsebaomo AP. Vernier acuity, crowding and cortical magnification. Vision Res. 1985;25:963-977. 